\title{
EVALUACIÓN DE UN PROGRAMA PILOTO PARA DESARROLLAR LA CONDUCTA PROSOCIAL EN NIÑOS DE EDAD ESCOLAR
}

\author{
Jennifer Stefania Herrera-Lozano y Bertha Musi Lechuga \\ Universidad Autónoma de Ciudad Juárez \\ México
}

\begin{abstract}
RESUMEN
El presente estudio fue un pilotaje para la adaptación del programa JUEGO, con el propósito de incrementar la conducta prosocial de los niños participantes por medio de juegos cooperativos. Se desarrolló con 37 niños con edades comprendidas entre los ocho y 10 años, mediante un diseño cuasiexperimental pre-post de un solo grupo, muestreo por conveniencia con variables cuantitativas y cualitativas. Entre los resultados se destaca que hubo un incremento reportado por los niños en la conducta prosocial y una disminución estadísticamente significativa en la conducta agresiva $p=0.024$ y en la pelea $p=0.018$. Se concluye que es posible incrementar la conducta prosocial y disminuir las conductas agresivas que inciden en el acoso escolar con la implementación del programa basado en juegos cooperativos.
\end{abstract}

Palabras Clave:

conducta prosocial, conducta agresiva, acoso escolar, edad escolar y juegos cooperativos.

\section{EVALUATION OF A PILOT PROGRAM TO DEVELOP PROSOCIAL BEHAVIOR IN SCHOOL-AGE CHILDREN}

\begin{abstract}
The present study was a pilot for the adaptation of the JUEGO program with the objective of increasing the prosocial behavior of the participating children through cooperative games. It was carried out with 37 children aged between 8 and 10 years, using a pre-post quasi-experimental design of a single group, convenience sampling with quantitative and qualitative variables. The results highlight that there was an increase reported by children in prosocial behavior and a statistically significant decrease in aggressive behavior $p=0.024$ and in the fight $p=0.018$. It is concluded that it is possible to increase the prosocial behavior and decrease the aggressive behaviors that affect bullying with the implementation of the program based on cooperative games.
\end{abstract}

Keywords:

prosocial behavior, aggressive behavior, bullying, school age, cooperative games

Bitácora del ARtículo:

| Recibido: 14 de diciembre de 2019 | Aceptado: 8 de Junio de 2020 | Publicado en línea: Julio-Diciembre de 2020 | 
Artículo Empírico | Evaluación de un programa piloto para desarrollar la conducta... Herrera-Lozano $\mathcal{L} \mathcal{M}$ Musi-Lechuga

\title{
Autoría y Derechos de Propiedad Intelectual
}

\section{EVALUACIÓN DE UN PROGRAMA PILOTO PARA DESARROLLAR LA CONDUCTA PROSOCIAL EN NIÑOS DE EDAD ESCOLAR}

\author{
Jennifer Stefania Herrera-Lozano y Bertha Musi Lechuga \\ Universidad Autónoma de Ciudad Juárez \\ México
}

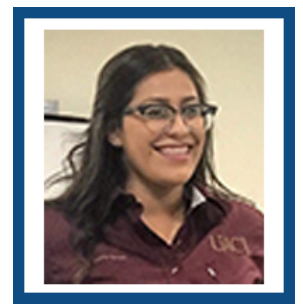

Jennifer Stefania Herrera-Lozano Universidad Autónoma de Ciudad Juárez

Correo: jen.ste.herrera@gmail.com

Licenciada en Educación por la Universidad Autónoma de Ciudad Juárez. Primer lugar en el Encuentro de Jóvenes Investigadores 2019 llevado a cabo en Chihuahua dentro del área de Humanidades y Ciencias de la Conducta. Autora de "Conductas y experiencias sociales en clase de niños escolarizados en el municipio de Sincelejo, Colombia".

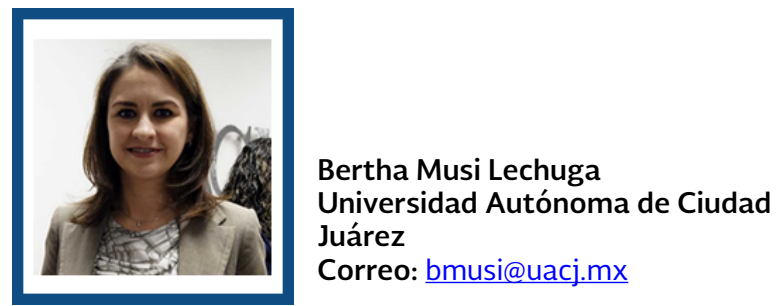

Doctora en Diseños de Investigación y Aplicaciones en Psicología y Salud; maestría en Diseños de Investigación y Aplicaciones en Psicología y Salud; máster internacional en Psicología Clínica. Psicoterapeuta, asesora psicoeducativa, orientadora vocacional y profesional. Profesora-investigadora, miembro del núcleo académico de la maestría en Psicología y, miembro del Comité Académico del Doctorado en Psicología de la UACJ.

\section{CONTRIBUCIÓN DE LAS AUTORAS}

Jennifer Stefania Herrera-Lozano concibió la redacción del documento a partir de la revisión bibliográfica, aplicó instrumentos, capturó los datos, hizo el análisis de datos y redactó los resultados preliminares. I Bertha Musi Lechuga asesoró la investigación por tiempo prolongado, haciendo revisiones a la redacción y coherencia del documento a partir de las reglas establecidas del Manual APA.

\section{AGRADECIMIENTOS}

Se agradece al personal docente y administrativo de la institución por la colaboración y las facilidades que brindaron para desarrollar esta investigación.

\section{DATOS DE FiLIACIÓN DE LAS AUtORAS}

Universidad Autónoma de Ciudad Juárez

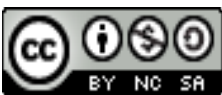

Copyright: (C) 2020 Herrera-Lozano, J.S. \& Musi-Lechuga, B.

Este es un artículo de acceso abierto distribuido bajo los términos de la licencia Creative Commons Reconocimiento-NoComercial 4.0 Internacional, por lo que su contenido gráfico y escrito se puede compartir, copiar y redistribuir total o parcialmente sin necesidad de permiso expreso de sus autoras con la única condición de que no se puede usar con fines directamente comerciales y los términos legales de cualquier trabajo derivado deben ser los mismos que se expresan en la presente declaración. La única condición es que se cite la fuente con referencia a la Revista Digital Internacional de Psicología y Ciencia Social y a sus autoras. 


\section{TABLA DE CONTENIDO}

INTRODUCCIÓN

MÉTOdo

Participantes, 359

Materiales, 360

Procedimiento, 360

Mediciones, 360

Reflexión por sesión, 360

Análisis estadísticos, 362

REsultados

Discusión

CONCLUSIONES 


\section{INTRODUCCIÓN}

H oy se ha destacado una normalización del acoso escolar como parte de la convivencia entre pares (Feldman, Donato y Wright, 2013; Herrera-Lozano, Vergara-Álvarez y Meza-Cueto, 2018), causando estragos en las diversas esferas del desarroIlo social, cognitivo y emocional (Popp, Peguero, Day y Kahle, 2014), a lo que diversos autores han marcado un amplio interés en la investigación de programas que favorezcan la promoción de conductas prosociales en busca de la inhibición del acoso escolar en edades tempranas (Mestre, Samper, Tur, Cortés y Nácher, 2006).

Se ha encontrado que al lograr una conducta prosocial dentro de grupos se mejoran los climas sociales generando empatía cognitiva y emocional entre ellos (Dovidio y Bandfield, 2015), de modo que cuando es aplicado en ambientes educativos se logra percibir una disminución de las conductas agresivas que generan el acoso escolar (Cajigas, Khan, Luzardo, Najson y Zalmalvide, 2004; Mestre et al., 2006; Redondo, Rueda y Amado, 2013). Entre los factores que determinan la conducta prosocial más mencionados por la literatura se encuentran la biología, la cultura, la familia, la crianza, la escuela, los rasgos de personalidad y los situacionales, ante lo que se debe considerar una interrelación entre éstos para lograr una eficacia en los programas que tengan como objetivo la conducta prosocial (Garaigordobil, 2017; Lemos, 2009; Mestre, 2014).

Con el transcurso de los años diversas investigaciones han contemplado que los estudios que incentivan la conducta prosocial tienden a generar beneficios que favorecen todas las esferas del desarrollo en edades tempranas (Garaigordobil 1995; Marín, 2010; Martela y Ryan, 2016), dotando de habilidades al individuo que facilitan su integración mediante conductas como compartir, ayudar, consolar o informar, que permiten una aceptación dentro de grupos sociales (Jensen, 2016). El enfoque de la conducta prosocial como estrategia para mejorar la convivencia escolar ha ayudado a hacer énfasis en la promoción de conductas positivas potencializando el bienestar individual y social (Redondo e Inglés, 2009), que permite disminuir el riesgo de generar acoso escolar (Fernández-Daza, 2016).

Las líneas de investigación centradas en el desarroIlo de la conducta prosocial en edades escolares coinciden en que esta conducta es explicada desde la teoría del aprendizaje social (Bandura, 1971) como un comportamiento aprendido por medio del modelamiento (Hogg y Vaughan, 2010). Partiendo de la interacción social, donde entran en contacto los componentes cognitivos con las situaciones a que se presente el individuo, ya sea en el contexto social, escolar o familiar (Méndez, Mendoza, Rodríguez y García, 2010) mediante la observación imitativa de figuras sociales (Schunk, 2012), por lo que al considerar la conducta prosocial se logra un efecto de modelaje en las conductas del grupo desatando una línea de beneficios en frecuencia, comenzando por facilitación del desarrollo integral del individuo, seguido por lograr efectos positivos en el clima social, que en conjunto mejoran la convivencia escolar (Carrasco y Trianes, 2010; Schoeps, Mónaco, Cotolí y Montoya-Castilla, 2020). Al mejorar el clima social positivo en edades escolares se favorece la percepción de reconocimiento, sentido de pertinencia y respeto mutuo entre pares (INEE, 2014) que se generan en el entorno escolar y son facilitadores del desarrollo social (Cerezo, Ruiz-Esteban, Sánchez y Arense, 2018).

Respecto a la mejora de los climas sociales en edades tempranas, es necesario considerar los juegos como una parte fundamental del desarrollo integral del ser humano y optarla como una estrategia viable para generar aprendizajes a partir de la experiencia (Osornio-Callejas, 2016) facilitando las posibilidades de experimentación (Garaigordobil, 1995; Espejel y Góngora, 2017), por lo que al considerar juegos cooperativos como una estrategia de aprendizaje es posible lograr mejoras en la conducta prosocial y los climas escolares son viables para el establecimiento de lazos afectivos entre pares (Mega y Liesa, 2017; Schoeps et al., 2020).

Si al considerar el juego como una estrategia para mejorar la conducta prosocial se logra un efecto dominó en la aceptación entre pares, un alto nivel de participación en el grupo y una atención positiva hacia los demás (Redondo e Inglés, 2009), es porque radica con fuerte influencia el desarrollo psicológico, emocional y social dentro de la infancia (Damián-Díaz, 2016). Entre las intervenciones que lo han logrado se destaca el programa JUEGO de Garaigordobil (1995) creado en España que está basado en la implementación de juegos cooperativos y creativos. Este programa se compone de cinco propuestas que abarcan desde preescolar hasta secundaria (Garaigordobil y Fagoaga-Azumendi, 2006), de los cuales este estudio se enfoca en el diseñado para tercer y cuarto grados de primaria. Este programa tiene como objetivo general potenciar el desarrollo integral de los niños que no presentan dificultades en su crecimiento, incidiendo sobre todo en diversos aspectos 
socioemocionales y en el desarrollo de la creatividad. Está compuesto por 60 juegos divididos en cinco tipos de juego; comunicación y cohesión grupal, ayuda y confianza, creatividad grupal, y cooperación y expresión emocional. El diseño de la intervención se hizo en 20 sesiones anuales que fueron aplicadas de modo semanal. La evaluación se efectuó con la técnica experimental de evaluación de la conducta altruista: El dilema del prisionero; Escala de comportamiento asertivo para niños; El juego de las siluetas; La escala de sensibilidad social, Batería de socialización y un listado de adjetivos. El estudio empírico que se hizo de este programa fue en 154 niños en edades entre los ocho y 11 años. La implementación fue desarrollada por el docente a cargo del grupo, mientras que la evaluación la hizo un observador que colaboró, obteniendo como resultado un notable descenso de conductas agresivas, un incremento de las conductas altruistas al igual que en el autoconcepto global y en la comunicación positiva intergrupal.

Asimismo, Garaigordobil y Berrueco (2007) implementaron el programa JUEGO 4-6 años para que el programa de intervención basado en el juego amistoso, de ayuda y cooperación, estimulara el desarrollo infantil en edades tempranas. El estudio se efectuó con 86 participante con edades de cinco y seis años contemplando grupo control y experimental. Se desarrolló con una metodología experimental con medidas repetidas pretest-postest. La implementación se hizo durante 75 minutos cada semana durante todo el curso escolar. La estructura de las sesiones se dio en tres fases: apertura, desarrollo de la secuencia de juego y cierre. Los instrumentos de evaluación que utilizaron fueron el test de resoluciones de problemas interpersonales, una evaluación del altruismo, el test breve de inteligencia, el cuestionario de madurez neuropsicológica infantil y la escala observacional del desarrollo, obteniendo como resultado un incremento significativo de la capacidad para buscar soluciones prosociales a problemas de interacción, al igual que aumentó la inteligencia emocional, confirmando la viabilidad de los juegos cooperativos para favorecer el desarrollo social y cognitivo.

Por otra parte, en Yucatán Osés, Duarte y Pinto (2016) desarrollaron una adaptación del programa JUEGO que está dirigido a niños de 10 a 12 años. La implementación se efectuó en 89 niños con edades de entre 11 y 15 años. Entre las características de aplicación destaca que pretendía fomentar la socialización, comunicación, expresión de sentimientos y cohesión grupal que fomentan conductas como compartir, ayudar, tolerar, cooperar que faciliten el desarrollo integral. Se llevó a cabo en 20 sesiones semanales donde se efectuaron se- siones de retroalimentación entre alumnos y docente. Las mediciones se hicieron mediante la escala de Comportamiento Asertivo para Niños y el Cuestionario de Estrategias Cognitivas de Resolución de Situaciones Sociales. Se realizó en cinco fases: 1) diagnóstico; 2) adaptación del programa; 3 ) entrenamiento; 4) aplicación del programa, y 5) evaluación. El resultado fue un impacto favorable en la conducta asertiva y en la disminución de conductas pasivas. Se concluyó la importancia del fomento de juegos cooperativos para la promoción de estrategias cognitivas para la resolución de situaciones sociales en los niños.

A partir de estos estudios se determina que el programa JUEGO puede ser adaptado para incidir en conductas que favorecen el desarrollo integral de los niños, debido a que esta gama de programas tiene como finalidad potenciar el desarrollo integral de niños sin dificultades de crecimiento e incorporar a niños con problemas de integración (León, 2008), por lo que a pesar de las valiosas contribuciones que se han indicado, hay una carente necesidad en adaptar el programa JUEGO para incrementar la conducta prosocial de los niños participantes por medio de juegos cooperativos, proponiendo siete hipótesis; en concreto, se hipotetiza que el programa generará cambios: $\mathrm{H} 1$ incrementará la conducta prosocial de los participantes después de concluir la intervención; en consecuencia, se pretende que este estudio proporcione respuesta al resto de las siguientes hipótesis planteadas; $\mathrm{H} 2$ reducirá la conducta agresiva después de efectuar la intervención con los participantes; H3 disminuirá la intimidación después de hacer la intervención con los participantes; H4 reducirá la burla después de desarrollar la intervención con los participantes; H5 reducirá la pelea después de efectuar la intervención con los participantes; H6 disminuirá la actitud personal hacia la violencia después de la intervención, y H7 se mantendrá la influencia externa de los participantes luego de la intervención.

\section{MÉTodo}

Este estudio tuvo como objetivo incrementar la conducta prosocial de los niños participantes por medio de juegos cooperativos y se basó en un diseño cuasiexperimental con variables cuantitativas y cualitativas.

\section{Participantes}

Mediante un diseño cuasiexperimental pre-post de un solo grupo se aplicó el programa en una muestra por conveniencia compuesta por 37 estudiantes con edades comprendidas entre ocho y 10 años, pertenecientes a 
cuarto grado de una escuela primaria pública. Presentando una media de edad de 9.24, siendo $37.83 \%$ niños y $62.17 \%$ niñas. Los criterios de inclusión para la participación de los menores fue que tuvieran una edad entre ocho y 10 años. Tener el consentimiento firmado por los padres o tutores. Los criterios de exclusión fueron que tuvieran una edad fuera de las establecidas, o en su defecto que los padres o tutores no autorizaran el consentimiento de participación.

\section{Materiales}

El programa JUEGO para niños de ocho a 10 años fue diseñado en España por Garaigordobil (1995) y es parte de una gama de varios programas que abarcan desde preescolar hasta la secundaria. JUEGO está basado en juegos cooperativos y creativos que busca potenciar el desarroIlo integral de los niños que no presentan dificultades en su crecimiento, incidiendo sobre todo en diversos aspectos socioemocionales y en el desarrollo de la creatividad. Está diseñado con 60 juegos para aplicarse cada semana durante 20 sesiones en el transcurso del ciclo escolar, con una duración de 90 minutos por sesión. Estos juegos están divididos en cinco categorías según el objetivo que persiguen (juegos de comunicación y cohesión grupal, de ayuda-confianza, de creatividad grupal, de cooperación y juegos de expresión emocional).

\section{Procedimiento}

En principio se contactó a la doctora Garaigordobil para hacer una adaptación e implementación del programa JUEGO en población mexicana. Una vez conseguido, se desarrollaron cinco fases: 1) propuesta de intervención; 2) gestión institucional; 3) evaluación inicial de los participantes; 4) implementación de la intervención, y 5) evaluación final para medir el impacto del programa.

La fase de propuesta de intervención se enfocó en adaptar el programa considerando las características específicas de la población y sentido cultural de algunos juegos. Para efectuar los cambios se tomaron los juegos de comunicación y cohesión grupal, el de ayuda-confianza y los de cooperación que, según la clasificación propuesta por la autora, favorecen la adaptación del programa específicamente para la conducta prosocial. Es importante aclarar que las sesiones fueron reestructuradas respecto a la secuencia de juegos, debido a que se tomaron en específico los que contribuirían de manera directa al objetivo del estudio. Luego se hizo la segunda fase de gestión institucional, donde se consideraron los aspectos éticos, los cuales incluyen el consentimiento informado de padres o tutores, anonimato de los datos, participación voluntaria y la libertad de abandonar la participación cuando lo deseen. Al concluir la fase dos se procedió a la fase tres, donde en una sesión se hizo la evaluación inicial de los participantes con apoyo de la escala de agresión entre pares; el instrumento se aplicó de manera grupal. En la fase cuatro se implementó el programa que constaba de una sesión de capacitación al docente frente a grupo y 15 sesiones basadas en juegos cooperativos para los niños (tabla 1). Estas sesiones en general fueron iniciadas con un establecimiento de reglamento para juegos, una conceptualización de la conducta prosocial, seguida de juegos cooperativos y el desarrollo de una reflexión acerca de los comportamientos presentados durante la sesión. Es necesario destacar que los docentes frente a grupo tuvieron una participación activa dentro del programa. Por último se efectuó la fase cinco, que fue la implementación, de manera grupal, de la escala de agresión entre pares.

\section{Mediciones}

\section{Escala de agresión entre pares [EAP]}

El instrumento utilizado fue la escala de agresión entre pares (EAP) de Cajigas et al. (2004). El instrumento está compuesto por 35 ítems y la medición se hace mediante escalas tipo Likert. A continuación se muestran las escalas y subescalas con que cuenta el instrumento. La escala de actitud personal hacia la violencia está compuesta por la actitud facilitadora que abarca los primeros cuatro ítems y por la actitud falta de manejo de los ítems 5 y 6 . La escala de conductas prosocial de los ítems 7 a 11. La escala de conductas agresivas está compuesta por las subescalas de pelea de los ítems 12 a 17; la subescala de intimidación se encuentra entre los ítems 18 a 21, y la burla por los ítems 22 a 24. Por último está la escala de influencias externas compuesta por la influencia de amigos en los ítems 25 a 31, y la influencia de adultos de los ítems 32 a 35.

\section{Reflexión por sesión}

La reflexión por sesión se efectuó al finalizar cada una de las sesiones en un formato donde se solicitaban el nombre y número de sesión. Los niños tenían la instrucción de describir cómo fue la conducta prosocial y las conductas agresivas durante cada una de las sesiones. El análisis de cada sesión se hizo con una sistematización de los conceptos donde se identificaba la presencia o ausencia de las conductas en cada una de las reflexiones. En el caso de la conducta prosocial se consideraron los reportes de ayudar, cooperar, compartir y prestar, mientras que en las conductas agresivas se consideraron los reportes de peleas, enojos, golpes, empujones, jalar el cabello, portarse mal con los demás y no respetar a otros. 
Tabla 1.

Plan de intervención.

\begin{tabular}{|c|c|c|c|c|c|}
\hline Sesión & ETAPA & Овjetivo & ACTIVIDADES & $\begin{array}{l}\text { TÉCNICAS DE } \\
\text { INTERVENCIÓN }\end{array}$ & $\begin{array}{l}\text { INSTRUMENTOS DE } \\
\text { REGISTRO }\end{array}$ \\
\hline Docente & $\begin{array}{l}\text { Capacitación } \\
\text { docente }\end{array}$ & $\begin{array}{l}\text { Capacitar al docente respecto a la im- } \\
\text { plementación del programa }\end{array}$ & $\begin{array}{l}\text {-Psicoeducación } \\
\text {-Explicación de juegos }\end{array}$ & & \\
\hline Ev & $\begin{array}{l}\text { Evaluación } \\
\text { diagnóstica } \\
\text { inicial. }\end{array}$ & $\begin{array}{l}\text { Identificar las conductas prosociales, } \\
\text { las conductas agresivas, la actitud faci- } \\
\text { litadora hacia la violencia y relaciones } \\
\text { interpersonales que presentan los parti- } \\
\text { cipantes antes de la intervención. }\end{array}$ & $\begin{array}{l}\text { Aplicación de instrumen- } \\
\text { tos. }\end{array}$ & $\begin{array}{l}\text { Aplicación de instrumen- } \\
\text { tos. }\end{array}$ & $\begin{array}{l}\text { Escala de agresión entre } \\
\text { pares }(E A P) \text {. }\end{array}$ \\
\hline
\end{tabular}

Apertura del programa: consigna de introducción al programa de juego. ción

9
Desarrollar la cooperación y la comunicación grupal.
-Creación del reglamen-

-Cesta de frutas.

-Mensajes misteriosos.

-Las burbujas.

- Me gusta de ti.

Desarrollar la ayuda, la confianza y la Pilotando aviones. comunicación grupal.

-El secreto.

-Busca tu pareja.

Desarrollar la cooperación y la comunicación grupal.

-Dibujo en colabora-

ción.

Desarrollar las conductas de ayuda y -El cerdito.

confianza.

-El amigo mudo.

Desarrollar las relaciones de ayuda y la -Cadeneta perseguidocooperación corporal.

ra. -Gatos y perros. Desarrollar las conductas de ayuda, confianza y cooperación.

masa.

- Pescar con las manos.

-Fábrica de juguetes.

Desarrollar la comunicación.

-Don Juan Tenorio ha muerto.

Desarrollar las conductas de ayuda, -Mensaje para... confianza y la comunicación. -Siluetas amistosas

Desarrollar las conductas de ayuda, -El ciempiés. confianza y cooperación.

-Abrazados.

Desarrollar la cooperación corporal y -Lápiz en la botella. las relaciones de ayuda. -Dictado de dibujos.

Desarrollar las conductas de ayuda, -Pelota en cadena. confianza y cooperación.

-El tragapeces.

Desarrollar la cooperación y las con- -Los elefantes. ductas de ayuda. -Rojo o azul.

Desarrollar la cooperación y la con- -Los camellos. Desarrollar la cooperación y la con-
fianza. -El guiño.

Desarrollar las relaciones de ayuda y la -El tesoro secreto del pirata cooperación.

barbaroja.

-Los dragones escondidos.

Identificar las conductas prosociales, Evaluación de las conductas agresivas, la actitud facila interven- litadora hacia la violencia y relaciones
Interven-

-Juegos de comunicación y cohesión grupal.

-Juegos de cooperación

Reflexión de sesión.

$-4$

-Con las manos en la ción.
Aplicación de instrumen- Aplicación de instrumen- Escala de agresión entre tos.
-Juegos de comunicación y cohesión grupal.

Reflexión de sesión. -Juegos de cooperación

-Juegos de comunicación y -Juegos de cooperación

-Juegos de comunicación y cohesión grupal. -Juegos de cooperación

Reflexión de sesión.

-Juegos de comunicación y cohesión grupal. -Juegos de cooperación

Reflexión de sesión.

-Juegos de comunicación y cohesión grupal.

Reflexión de sesión. -Juegos de cooperación

-Juegos de comunicación y

cohesión grupal.

Reflexión de sesión.

-Juegos de comunicación y cohesión grupal.

Reflexión de sesión. -Juegos de cooperación

-Juegos de comunicación y cohesión grupal.

Reflexión de sesión. -Juegos de cooperación

-Juegos de comunicación y

cohesión grupal.

Reflexión de sesión. -Juegos de cooperación

-Juegos de comunicación y cohesión grupal. -Juegos de cooperación

Reflexión de sesión

-Juegos de comunicación y cohesión grupal.

Reflexión de sesión. -Juegos de cooperación

-Juegos de comunicación y cohesión grupal.

Reflexión de sesión. -Juegos de cooperación

-Juegos de comunicación y cohesión grupal.

Reflexión de sesión. -Juegos de cooperación
-Juegos de cooperación pares (EAP). interpersonales que presentan los participantes antes de la intervención. 


\section{Análisis estadísticos}

Los análisis estadísticos que se hicieron fueron una prueba T para muestras relacionadas en cada una de las subescalas de la escala de agresión entre pares [AEP], que obtuvo un alfa de Cronbach de 0.882 , mientras que con lo recabado de las reflexiones con una sistematización de la conducta prosocial y conductas agresivas que fueron percibidas por los niños.

\section{Resultados}

En la tabla 2 se muestran los cambios obtenidos a partir de la intervención en cada una de las parejas de pretest y postest. Es evidente que se lograron cambios estadísticamente significativos en la pelea, la conducta agresiva y la conducta prosocial.

Tabla 2.

Estadísticos descriptivos de los resultados de la escala de agresión entre pares pre-post

\begin{tabular}{|c|c|c|c|c|}
\hline & & Media & $\begin{array}{l}\text { DESVIACIÓN } \\
\text { ESTÁNDAR }\end{array}$ & $\begin{array}{c}\text { Sig } \\
\text { (BILATERAL) }\end{array}$ \\
\hline \multirow{2}{*}{$\begin{array}{l}\text { Actitud } \\
\text { personal hacia } \\
\text { la violencia }\end{array}$} & Pretest & 13.1351 & 4.96187 & 0.584 \\
\hline & Postest & 12.7027 & 4.64797 & \\
\hline \multirow{2}{*}{$\begin{array}{l}\text { Actitud } \\
\text { facilitadora }\end{array}$} & Pretest & 9.0811 & 3.96778 & 0.248 \\
\hline & Postest & 8.3784 & 3.22621 & \\
\hline \multirow{2}{*}{$\begin{array}{l}\text { Actitud de } \\
\text { manejo }\end{array}$} & Pretest & 4.0541 & 2.2104 & 0.617 \\
\hline & Postest & 4.3243 & 2.79935 & \\
\hline \multirow{2}{*}{$\begin{array}{l}\text { Conducta } \\
\text { prosocial }\end{array}$} & Pretest & 16.6757 & 4.5829 & 0.008 \\
\hline & Postest & 15.027 & 4.73454 & \\
\hline \multirow{2}{*}{$\begin{array}{l}\text { Conductas } \\
\text { agresivas }\end{array}$} & Pretest & 24.1892 & 10.0162 & 0.024 \\
\hline & Postest & 21.0541 & 7.63015 & \\
\hline \multirow{2}{*}{ Pelea } & Pretest & 11.8108 & 5.44588 & 0.018 \\
\hline & Postest & 10.1351 & 4.13093 & \\
\hline \multirow{2}{*}{ Intimidación } & Pretest & 7.2973 & 2.8271 & 0.08 \\
\hline & Postest & 6.4595 & 2.65199 & \\
\hline \multirow{2}{*}{ Burla } & Pretest & 5.0811 & 2.85169 & 0.175 \\
\hline & Postest & 4.4595 & 1.87964 & \\
\hline \multirow{2}{*}{$\begin{array}{l}\text { Influencia } \\
\text { externa de } \\
\text { amigos }\end{array}$} & Pretest & 10.5676 & 3.03261 & 1 \\
\hline & Postest & 10.5676 & 2.89195 & \\
\hline \multirow{2}{*}{$\begin{array}{l}\text { Influencia } \\
\text { externa de } \\
\text { adultos }\end{array}$} & Pretest & 7.4054 & 2.42051 & 0.314 \\
\hline & Postest & 6.8919 & 2.36624 & \\
\hline
\end{tabular}

En la figura 1 se ilustra la evolución de las conductas agresivas y prosociales percibidas por los niños, encontrándose que la conducta prosocial obtuvo una media de 0.98 en el desarrollo de las sesiones, de las cuales 22 se manejaron por arriba de la media, mientras que en la conducta agresiva se encontró una media de 0.089 , donde de las 15 sesiones implementadas 10 de ellas se mantuvieron por debajo de la media de violencia reportada por los niños. En las últimas seis sesiones se observa que se logró una estabilidad de conducta prosocial. Asimismo es evidente que la sesión más agresiva que se presentó fue la 6 con una puntuación de 0.384 .

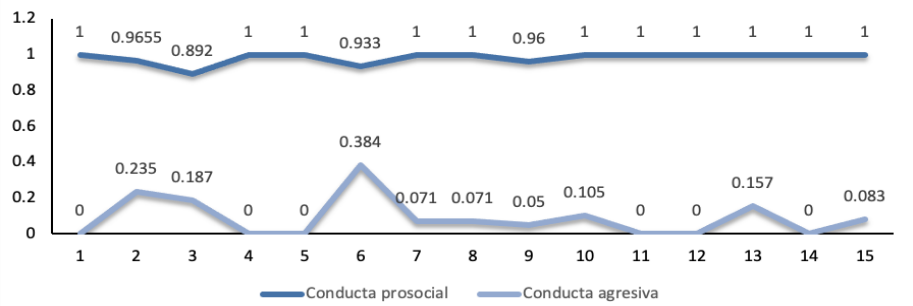

Figura 1.

Evolución de la conducta prosocial y conducta agresiva por sesión.

\section{Discusión}

Este estudio se efectuó como un pilotaje de la adaptación del programa JUEGO y tuvo como objetivo incrementar la conducta prosocial de los niños participantes por medio de juegos cooperativos. Respecto a las hipótesis, la $\mathrm{H} 1$ planteaba incrementar la conducta prosocial de los participantes; fue confirmada mediante la función inhibidora de la conducta ante conductas agresivas (Carrasco y Trianes, 2010; Garaigordobil, 2003; Mestre et al., 2006); esto quedó demostrado en la figura 1, donde se muestra que en $90 \%$ de las sesiones logró una completa conducta prosocial, mientras que la conducta agresiva se presentó debajo de la media o sin presencia. Sin embargo, en los datos que se muestran en la tabla 1 no se indica un incremento. Este hallazgo pudiese ser atribuido a la incertidumbre o temor que manifestaron los niños ante una deseabilidad social en la evaluación inicial; dicha deseabilidad es definida como una distorsión de la realidad por no quedar mal o cumplir con los estándares sociales deseados (Enríquez y Domínguez, 2010). Con el transcurso de las sesiones esta postura fue cambiando en los participantes hasta que se logró establecer una relación de confianza.

Por otra parte, atendiendo a lo planteado en la $\mathrm{H} 2$, que mencionaba que reducirá la conducta agresiva después de efectuar la intervención con los participantes, se encuentra que fue cumplida logrando una disminu- 
ción estadísticamente significativa. Es importante hacer hincapié en la sesión 6 que presentó un incremento de agresividad en comparación con el resto de las sesiones: esto es atribuido al diseño que tuvo esta sesión porque los tres juegos que lo componían llevaban algún tipo de contacto físico entre los participantes y tornaba las situaciones para jugar brusco de nuevo. De igual manera se logró lo planteado en la $\mathrm{H} 3$ respecto a la disminución de intimidación entre los participantes; la H4 se cumplió porque se evidenció una disminución luego de la intervención. La H5, que planteaba la disminución pelea, fue cumplida con cambios estadísticamente significativos. lo cual es coherente con el factor inhibidor que hay entre el aumento que se encontró en la conducta prosocial y la disminución de las conductas agresivas, situándose como alternativa en la presencia de estas conductas (Redondo, Rueda y Amado, 2013).

Existe una correlación entre la actitud personal hacia la violencia y las conductas agresivas porque esta actitud es un precursor a la generación de actos violentos (Ortiz-Tallo, 2014). Esto corresponde a la H6, donde se esperaba disminuir la actitud personal hacia la violencia después de la intervención, lo que se logró sin obtener una diferencia significativa. Por último, se cumplió lo establecido en la $\mathrm{H} 7$ referente a la influencia externa, obteniendo que la influencia de amigos permaneció intacta, pero la influencia externa de adultos disminuyó después de la intervención. Este fenómeno es atribuido a que se ha encontrado que la conducta prosocial hace distinciones en la cercanía de las personas receptoras de ayuda, es decir, no se desarrolla un proceso igual entre los amigos y los adultos pertenecientes a su familia (Mestre, 2014; Mesurado, 2014).

\section{Conclusiones}

Es necesario considerar una serie de limitaciones importantes que fueron encontradas en este estudio. La dificultad presentada en la evaluación cuantitativa de la conducta prosocial se debe a no haber sido considerada la deseabilidad social. Entre las consideraciones que se hacen se destaca el involucrar un poco más la participación de padres. En tercer lugar se determinó la falta de consideración de días inhábiles, ante los cuales fue necesario hacer modificaciones en los tiempos planeados y la falta de un psicopedagogo que diera acompañamiento y apoyo durante cada una de las sesiones.

Por su parte, este estudio destaca la relevancia de este tipo de investigaciones, que permiten identificar las deficiencias ante las que se puede enfrentar este tipo de intervenciones para que puedan ser mejoradas. Ante los hallazgos identificados en este pilotaje se considera de vital importancia tener un establecimiento de confianza y rapport previo a las evaluaciones. A su vez, se encontró que la evaluación quedó un poco corta para alcanzar a medir por completo la conducta prosocial, por lo que es necesario complementar las evaluaciones que se hacen para obtener resultados más enriquecedores y completos que permitan tener una perspectiva más amplia de la efectividad del programa.

En perspectiva, este estudio de pilotaje ofrece información enriquecedora y muestra áreas de oportunidad para la mejora y lograr un mayor impacto de la adaptación de programa. Entre estos hallazgos, se sugiere una mejora en el diseño de la sesión 6, donde se presentaron juegos que incluían contacto físico para evitar que se despegue la agresividad como se mostró en la figura 1.

De manera más general se hizo evidente considerar la implementación que busque prevenir conductas negativas en los ambientes educativos para lograr un clima social escolar que potencialice el desarrollo integral desde edades tempranas. Ante esto, es necesario considerar las intervenciones donde se busque dotar a los niños de habilidades como la conducta prosocial que faciliten el trabajo cooperativo para prevenir las agresiones o acoso escolar, esto con la finalidad de optimizar las relaciones interpersonales que adquiere el menor durante su desarrollo (Collell y Escudé, 2011).

En este trabajo se ha analizado la función del juego cooperativo como una estrategia para incrementar la conducta prosocial. Se identificó la importancia de generar mecanismos que promuevan esta conducta para mejorar la convivencia escolar. Se establece que deben considerarse programas que favorezcan la conducta prosocial en los grados educativos de acuerdo con los intereses particulares que demanda la etapa del desarrollo. Se destaca la importancia de investigaciones que permitan mejorar las conductas sociales positivas como estrategia de reducción al acoso escolar enfocadas en el mejoramiento del clima social dentro de las instituciones. Por último, destaca la importancia de considerar estudios dirigidos a la creación o adaptación de instrumentos que faciliten la evaluación de la conducta prosocial de niños mexicanos en edades escolares desde perspectivas más amplias.

\section{Referencias}

Bandura, A. (1971). Social Learning Theory. New York: General Learning Press.

Cajigas, S. N., Khan E., Luzardo, M., Najson, S., \& Zalmalvide, G. (2004). Escala de agresión entre pares para adolescentes y principales resultados. Acción psicológica, 3(3), 173-186.

Carrasco, C., \& Trianes, M. V. (2010). Clima social, prosocialidad y 
violencia como predictores de inadaptación escolar en la primaria. European Journal of Education and Psychology, $3(2), 229-242$.

Cerezo, F., Ruiz-Esteban, C., Sánchez, L. C., \& Arense, G. J. J. (2018). Dimensions of parenting styles, social climate, and bullying victims in primary and secondary education. Psicothema, 1(30), 59-65.

Collell, J., \& Escudé, C. (2011). Conductes d'exclusió i maltractament entre iguals al parvulari. Ámbits de Psicopedagogia, (32), 37-40.

Damián-Díaz, M. G. M. (2016). Los juegos elegidos por niños en el colegio, en la casa y el favorito. Revista Digital Internacional de Psicología y Ciencia Social, 1(2), 58-74. http://dx.doi. org/10.22402/j.rdipycs.unam.2.1.2016.63.58-68.

Dovidio, J. F., \& Bandfield, J. C. (2015). Prosocial Behavior and Empathy. En J. D. Wright (ed.). International Encyclopedia of the Social and Behavioral Sciences, pp. 216-220. Oxford, England: Elsevier.

Enríquez, J. F J., \& Domínguez, E. A. C. (2010). Influencia de la deseabilidad social (DS) en reportes de capacitación. Psicología Iberoamericana, 1(18), 69-79.

Espejel, L. M. V., \& Góngora, C. E. A. (2017). Conducta prosocial. Propuesta de una intervención a través del cuento y el juego cooperativo. Revista de Estudios Clínicos e Investigación Psicológica, 7(14), 138-154.

Feldman, H. M., Donato, I., \& Wright, J. (2013). Bullying and Suicide. A Public Health Approach. Journal Adolescent Health, 53, S1-S3.

Fernández-Daza, M. (2016). Neuropsicología del acoso escolar. Función mediadora de la conducta prosocial. Revista Mexicana de Neurociencia, 6(17), 106-119.

Garaigordobil, L. M. (2003). Diseño y evaluación de un programa de intervención socioemocional para promover la conducta prosocial y prevenir la violencia. España: Fer Fotocomposición.

Garaigordobil, L. M., \& Fagoaga-Azumendi, J. M. (2006). El juego cooperativo para prevenir la violencia en los centros escolares. España: Ministerio de Educación y Ciencia.

Garaigordobil, M. (1995). Evaluación de una intervención psicológica en indicadores del desarrollo moral. Boletín de Psicología, 49, 69-86.

Garaigordobil, M. (2017). Conducta antisocial: conexión con bullying/cyberbullying y estrategias de resolución de conflictos. Psychosocial Intervention, 26(1), 47-54. doi: http://dx.doi.org/10.1016/j.psi.2015.12.002.

Garaigordobil, M., \& Berrueco, L. (2007). Efectos de un programa de intervención en niños de 5 a 6 años. Evaluación del cambio proactivo en factores conductuales y cognitivos del desarrollo. SUMMA Psicológica, 2(4), 3-19.

Herrera-Lozano, J. S., Vergara-Álvarez, M. L., \& Meza-Cueto, L. M. (2018). Conductas y experiencias sociales en clases de niños escolarizados en el municipio de Sincelejo, Colombia. Búsqueda, 5(21), 212-230. doi: 10.21892/01239813.423.

Hogg, M. A., \& Vaughan, G. M. (2010). Psicología social. España: Editorial Médica Panamericana.

Instituto Nacional para la Evaluación de la Educación [INEE] (2014). La convivencia escolar en las escuelas primarias de México. México: INEE.

Jensen, K. (2016). Prosociality. Current Biology, (26), R739-R755. Lemos, V. (2009). Evaluación de la eficacia de un programa para promover la conducta prosocial en niños en riesgo social por pobreza. En M. C. Richaurd de Minzi, yJ. E. Moreno (eds.). Recientes desarrollos iberoamericanos en investigación en ciencias del comportamiento, vol. 1, pp. 137-152. ISBN 987950-692-091-3. Buenos Aires: CIIPME-CONICET.

León, Z. E. (2008). Juegos cooperativos y creativos para grupo de niños de 4 a 6 años. Informació Psicológica, 94, 84-85.

Marín, E. J. C. (2010). Revisión teórica respecto a las conductas prosociales. Análisis para una reflexión. Psicogente, 13(24), 369-388.

Martela, F., \& Ryan, R. M. (2016). Prosocial behavior increases wellbeing and vitality even without contact with the beneficiary. Causal and behavioral evidence. Motivation and emotion, 40, 351-357. doi: $10.1007 / \mathrm{s} 11031-016-9552-\mathrm{z}$.

Mega, A. A. M., \& Liesa, O. M. (2017). El juego cooperativo como método para favorecer la inclusión y el desarrollo de conductas prosociales. Infancia, Educación y Aprendizaje, 2(3), 649-654.

Méndez, T. F. C. C., Mendoza, Á. C., Rodríguez, R. L. M., \& García, M. M. (2010). Vertientes. Revista Especializada en Ciencias de la Salud, 18(2), 9-16.

Mestre, M. V. (2014). Desarrollo prosocial: crianza y escuela. Revista Mexicana de Investigación en Psicología, 6(2), 115-134.

Mestre, V., Samper, P., Tur, A. M., Cortés, T., \& Nácher, J. (2006). Conducta prosocial y procesos psicológicos implicados. Un estudio longitudinal en la adolescencia. Revista Mexicana de Psicología, 23(2), 203-215.

Mesurado, B. (2014). Nuevas perspectivas en investigación sobre la conducta prosocial. La identificación del receptor de la ayuda y la motivación del agente de la conducta prosocial. Revista Mexicana de Investigación en Psicología, 6(2), 166-170.

Ortiz-Tallo, M. F. (2014). Fortalezas psicológicas, actitudes hacia la violencia y sexismo durante la educación sexual secundaria. Un estudio longitudinal. Tesis doctoral. Universidad de Málaga. Recuperada de https://riuma. uma.es/xmlui/handle/10630/7600.

Osés, B. R. M., Duarte, B. E., \& Pinto, L. M. L. (2016). Juegos cooperativos. Efectos en el comportamiento asertivo en niños de 6o. grado de escuelas públicas. Revista Electrónica de Investigación Educativa, 3(18), 177-186.

Osornio-Callejas, L. (2016). Juegos cooperativos como proyecto de intervención para establecer una mejora de convivencia escolar, paz y armonía. Descripción de una experiencia en una escuela telesecundaria de Aculco. Ra Ximhai, 3(12), 415-431.

Popp, A. M., Peguero, A. A., Day, K. R., \& Kahle, L. L. (2014). Gender, Bullying Victimization, and Education. Violence and Victims, 5(29), 1-14.

Redondo, P. J., \& Inglés, S. C. J. (2009). Conducta prosocial atribuciones causales y rendimiento académico en adolescentes. Colombia: CESMAG.

Redondo, P. J., Rueda, R. S., \& Amado, V. C. (2013). Conducta prosocial. Una alternativa a las conductas agresivas. Investigium Ire: Ciencias Sociales y Humanas, 1(4), 234-247.

Schoeps, K., Mónaco, E., Cotolí, A., \& Montoya-Castilla, I. (2020). The impact of peer attachment on prosocial behavior, emotional difficulties and conduct problems in adolescence. The mediating role of empathy. PLoS ONE,15(1). Recuperado de https://doi.org/10.1371/ journal.pone.0227627.

Schunk, D. H. (2012). Teorías del aprendizaje. México: Pearson. 


\section{Meta-Análisis del Artículo}

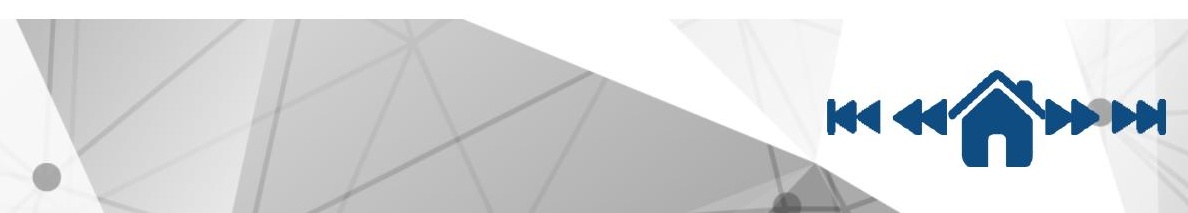


Revista Digital Internacional de Psicología y Ciencia Social $\mid$ Vol. 6 | SNúm. $2 \mid$ Julio-Diciembre 2020 | e-ISSN 2448-8119

\section{Dimensión Cuantitativa}

\section{Perfil de Evaluación entre pares}
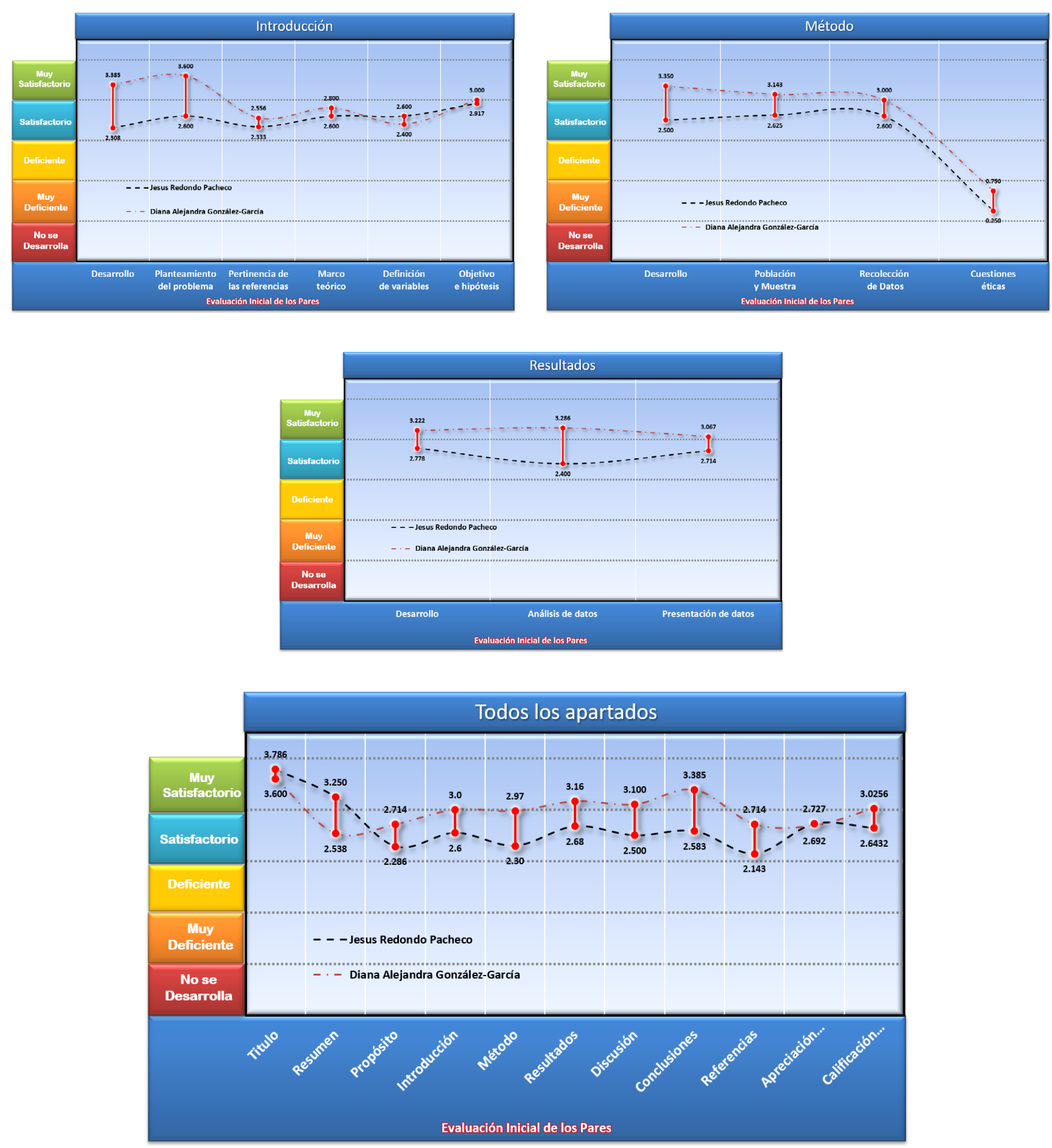


\section{Índice de Concordancia}

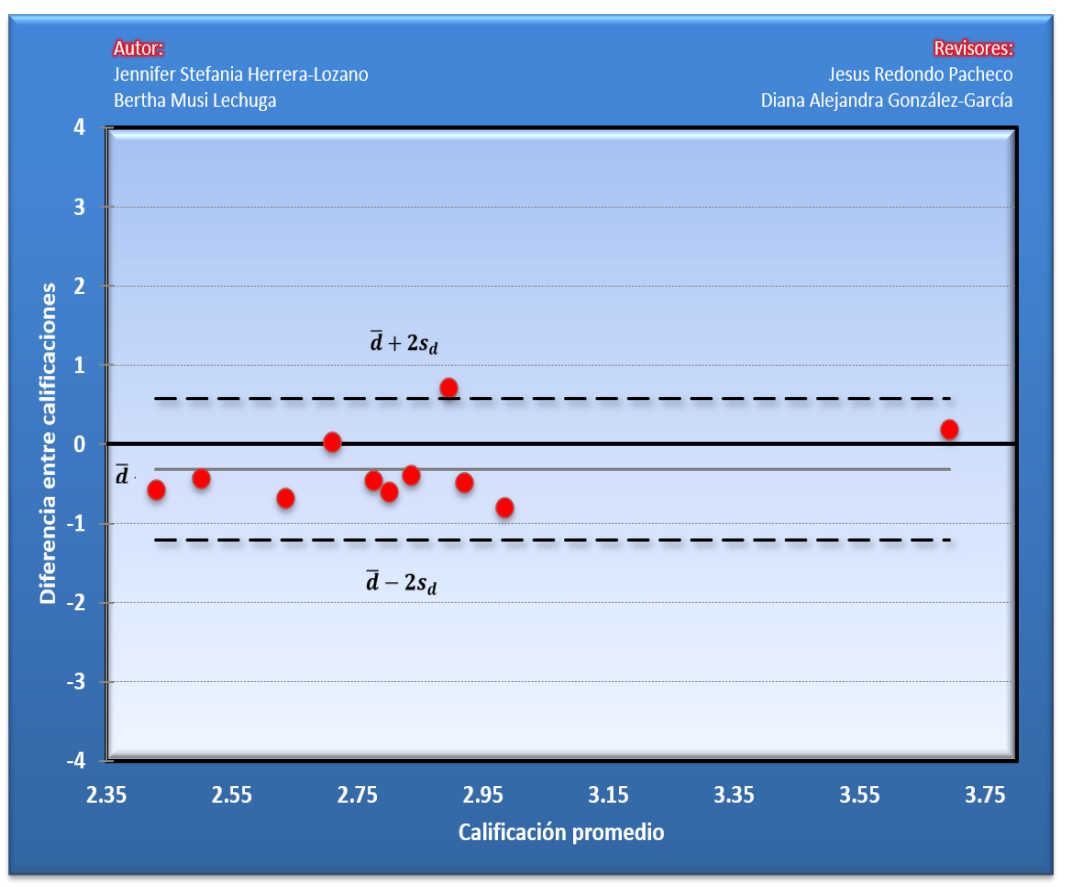

Índice de Acuerdo

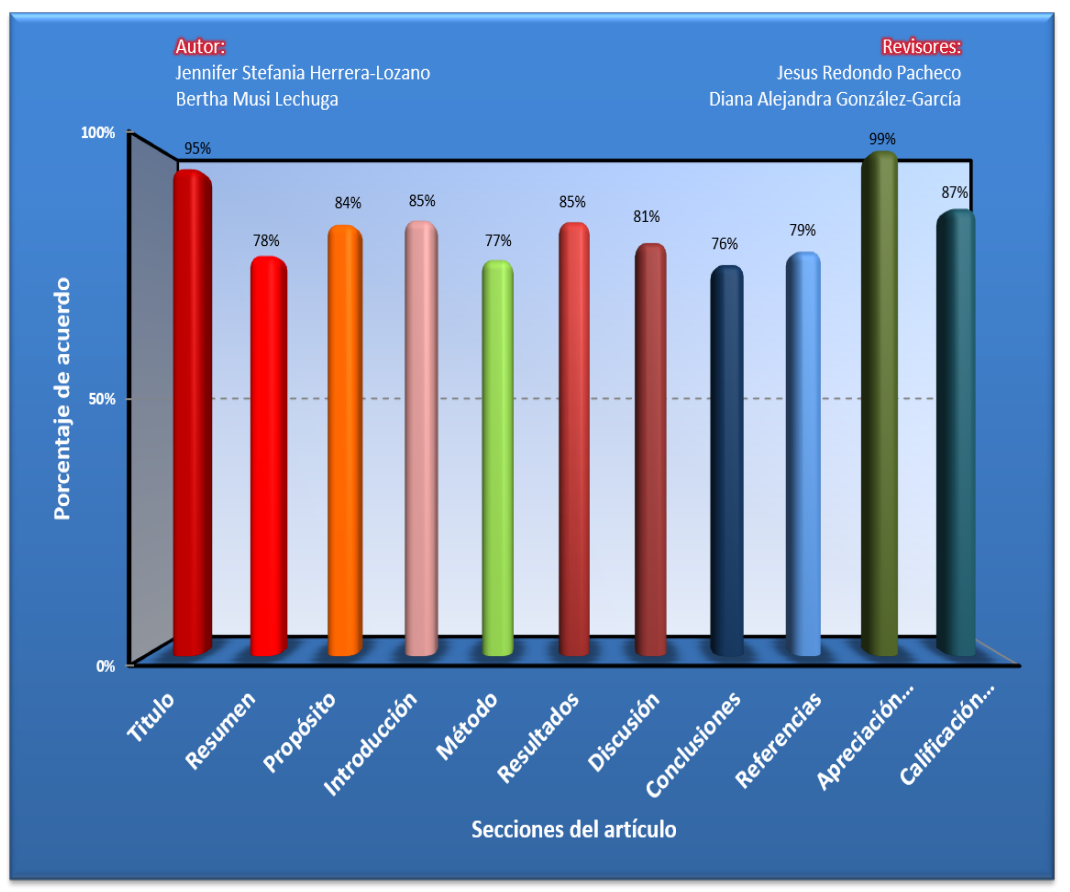




\begin{tabular}{|c|c|}
\hline Revisor 1 & Revisor 2 \\
\hline Jesus Redondo Pacheco & Diana Alejandra González-García \\
\hline \multicolumn{2}{|c|}{ Título/Autoría } \\
\hline $\begin{array}{l}\text { Sección relacionada con el título correcta según los cri- } \\
\text { terios de la revista }\end{array}$ & $\begin{array}{l}\text { El titulo es fácil de entender y explicativo. Considero } \\
\text { que no refleja el espíritu del estudio que es la adapta- } \\
\text { ción de una intervención española para aumentar la } \\
\text { conducta prosocial al contexto mexicano. }\end{array}$ \\
\hline \multicolumn{2}{|c|}{ Resumen } \\
\hline Se hacen algunas observaciones de forma en el documento & $\begin{array}{l}\text { La primera y segunda oración ponen en contexto el proble- } \\
\text { ma de investigación y el tercero enuncia propiamente el pro- } \\
\text { pósito. Respecto al método solamente explica que se adapto } \\
\text { a México. Se menciona que se utilizó la escala de Agresión } \\
\text { entre Pares y solamente menciona que existen "resultados } \\
\text { preliminares" no se mencionan promedios, tasas o estadís- } \\
\text { ticos que se utilizaron para el análisis. No se menciona una } \\
\text { conclusión sobre el estudio. Hay inconsistencia en los ver- } \\
\text { bos que se utilizan y desequilibrio entre la información que } \\
\text { se debe presentar en casa sección (hay más información so- } \\
\text { bre el contexto que sobre las conclusiones). }\end{array}$ \\
\hline \multicolumn{2}{|c|}{ Próposito del Estudio } \\
\hline $\begin{array}{l}\text { Se deben revisar varios aspectos relacionados con los } \\
\text { resultados y discusión que llevará a aclarar el propósito } \\
\text { del estudio }\end{array}$ & $\begin{array}{l}\text { Considero importante señalar que la intervención es una } \\
\text { adaptación de una intervención Española adaptada al con- } \\
\text { texto mexicano. Esta sección presenta un propósito y varias } \\
\text { hipótesis que se deben presentar o añadir en una oración } \\
\text { aparte. En ellas se especifica la dirección en que se modi- } \\
\text { ficarán cada una de las conductas agresivas a partir de la } \\
\text { intervención. Puede resumir todo en una sola oración. }\end{array}$ \\
\hline
\end{tabular}




\begin{tabular}{|c|c|}
\hline Revisor 1 & Revisor 2 \\
\hline \multicolumn{2}{|c|}{ Introducción } \\
\hline $\begin{array}{l}\text { Se deben considerar las observaciones hechas en el do- } \\
\text { cumento. }\end{array}$ & $\begin{array}{l}\text { La propuesta es interesante, aunque es una adaptación de } \\
\text { una intervención desarrollada en España, no es un Piloto } \\
\text { de una nueva intervención. Sería conveniente describir } \\
\text { dicha intervención (i.e., estudio de Garaigordobil, 1995), } \\
\text { con mucho detalle respecto los participantes, los compo- } \\
\text { nentes de la intervención (teoría en la que está basada), } \\
\text { instrumentos de evaluación, resultados considerar las im- } \\
\text { plicaciones y si los autores identificaron limitaciones. }\end{array}$ \\
\hline \multicolumn{2}{|c|}{ Método } \\
\hline $\begin{array}{l}\text { Se recomienda revisar las observaciones en estos apar- } \\
\text { tados ya que se presentan algunos déficits que deben } \\
\text { ser mejorados para ser publicado }\end{array}$ & $\begin{array}{l}\text { La sección de instrumentos parece parte del procedimien- } \\
\text { to. Debe presentarse antes del procedimiento. La sección } \\
\text { de materiales describe de manera escueta la intervención. } \\
\text { Se sugiere describirla con más detalle, tal vez en una sec- } \\
\text { ción independiente o como parte del procedimiento, in- } \\
\text { cluyendo una descripción de los componentes o módulos } \\
\text { que la integran, así como ejemplos de las actividades que } \\
\text { se realizan. Incluso puede señalar la distribución de las se- } \\
\text { siones en relación con los módulos. Haciendo énfasis en } \\
\text { cuales fueron las modificaciones/adaptaciones y justificar } \\
\text { por qué no se utilizaron los mismos instrumentos que en } \\
\text { España y la equivalencia con los instrumentos utilizados. } \\
\text { No se especifica como se analizarán las respuestas de la } \\
\text { reflexión que realizan los niños al final de cada sesión. }\end{array}$ \\
\hline \multicolumn{2}{|c|}{ Resultados } \\
\hline $\begin{array}{l}\text { Se recomienda revisar las observaciones debido a que } \\
\text { el programa piloto es fundamental y eje central en este } \\
\text { artículo pero no se evidencia en el trabajo ninguna de } \\
\text { las actividades o sesiones realizadas ni cómo se llevaron } \\
\text { a cabo. Éste es un problema importante del documento }\end{array}$ & $\begin{array}{l}\text { Puede resumir la Tabla } 1 \text { o solo presentar los estadísticos } \\
\text { sobre las diferencias significativas en el mismo texto sin } \\
\text { presentar la tabla. No se explica como se obtuvo la Figu- } \\
\text { ra 1, es pertinente pues parece más un procedimiento de } \\
\text { grupos focales para obtener la definición de las conductas } \\
\text { agresivas o prosociales. De ser así que se explique el proce- } \\
\text { dimiento para la obtención de la información y su análisis. } \\
\text { Hasta aquí el diseño del estudio parece un diseño mixto } \\
\text { por la introducción del análisis de las reflexiones. }\end{array}$ \\
\hline
\end{tabular}




\begin{tabular}{|c|c|}
\hline Revisor 1 & Revisor 2 \\
\hline \multicolumn{2}{|c|}{ Discusión } \\
\hline Se debe mejorar considerablemente este apartado & $\begin{array}{l}\text { Se presentan los resultados aunque no todos los resul- } \\
\text { tados se discuten o contrastan con la literatura. Con- } \\
\text { sidero que podría añadir biografía más reciente para } \\
\text { ayudar a discutir los resultados. }\end{array}$ \\
\hline \multicolumn{2}{|c|}{ Conclusiones } \\
\hline Revisar las observaciones hechas en el documento & $\begin{array}{l}\text { Se habla de que el pilotaje proveyó de información va- } \\
\text { liosa sobre como mejorarlo. No obstante, en la sección } \\
\text { de resultados no se mencionan cuales son los datos que } \\
\text { hacen evidentes las dificultades o deficiencias de la in- } \\
\text { tervención ni porqué se deben ajustar. Probablemente } \\
\text { es necesario reportar otros datos en la sección de re- } \\
\text { sultados. Algunas dificultades eran predecibles desde } \\
\text { el diseño de la investigación porque no se incluyeron } \\
\text { medidas equivalentes al estudio original. }\end{array}$ \\
\hline \multicolumn{2}{|c|}{ Referencias } \\
\hline Es un apartado con muchas deficiencias & $\begin{array}{l}\text { Existen diversos errores en el trabajo. Revisar con cui- } \\
\text { dado. }\end{array}$ \\
\hline
\end{tabular}

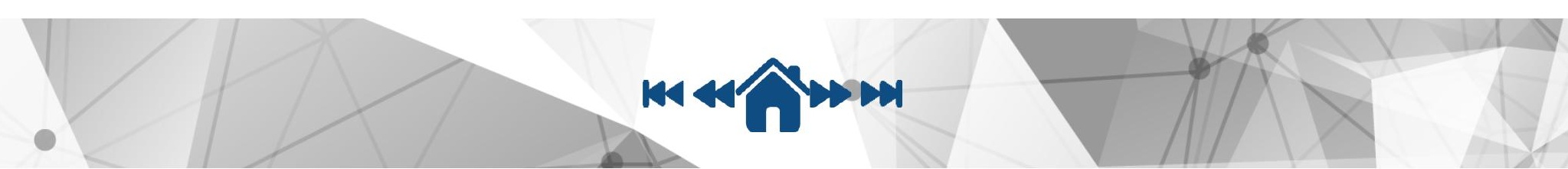




\section{Historia del Proceso}

\section{EDITORIAL}

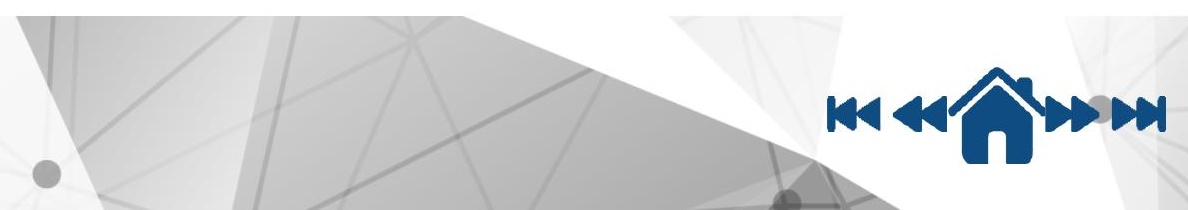

